\title{
Antibiotic Resistance Trends Among Ocular Pathogens in the US-Cumulative Results from the Antibiotic Resistance Monitoring in Ocular Microorganisms (ARMOR) Surveillance Study
}

Penny A Asbell ${ }^{1}$ and Christine M Sanfilippo ${ }^{2}$

1.Cornea Service and Refractive Surgery Center, Icahn School of Medicine at Mount Sinai, New York, New York, US; 2. Pharmaceutical Medical Affairs, Bausch + Lomb, Rochester, New York, US

A ntibiotic resistance among ocular pathogens is a public health concern. The multicenter, prospective Antibiotic Resistance Monitoring in Ocular micRoorganisms (ARMOR) study is an ongoing surveillance study designed to report on antibiotic resistance rates and trends among Staphylococcus aureus, coagulase-negative staphylococci (CoNs; includes Staphylococcus epidermidis), Streptococcus pneumoniae, Pseudomonas aeruginosa, and Haemophilus influenzae isolates from ocular infections. Results for more than 4,000 isolates collected from 2009 -2015 , representing 7 years of ARMOR, were recently presented. More than a third of $S$. aureus and almost half of all CoNS isolates were found to be resistant to methicillin. Staphylococcal isolates also showed high levels of multidrug resistance (resistance to $\geq 3$ antibacterial drug classes) with $76.4 \%$ and $73.7 \%$ of methicillin-resistant S. aureus (MRSA) and methicillin-resistant CoNS (MRCONS) isolates, respectively, demonstrating multidrug resistance. Resistance among S. pneumoniae was notable for azithromycin (36.8\%) and for penicillin (34.0\%), whereas P. aeruginosa and $\mathrm{H}$. influenzae were generally susceptible to the antibiotic classes tested. Longitudinal analyses demonstrated a small decrease in methicillin resistance among S. aureus over the 7-year study period, which may be a result of improved antibiotic stewardship. Continued surveillance of antibiotic resistance among ocular pathogens is warranted.

\section{Keywords}

ARMOR, surveillance study, antibiotic resistance, ocular infections, Staphylococcus aureus

Disclosure: Penny A Asbell is a consultant to Perrigo and Kurobe, and participates in advisory boards for Valeant/Bausch + Lomb. She is a speaker on continuing medical education topics at professional meetings for Vindico. Christine M Sanfilippo is an employee of Bausch + Lomb.

Acknowledgment: Medical writing assistance was provided by Katrina Mountfort and Michelle Dalton for Touch Medical Media, supported by Bausch + Lomb, a division of Valeant Pharmaceuticals International Inc.

Authorship: All named authors meet the International Committee of Medical Journal Editors (ICMJE) criteria for authorship of this manuscript, take responsibility for the integrity of the work as a whole, and have given final approval to the version to be published.

Open Access: This article is published under the Creative Commons Attribution Noncommercial License, which permits any noncommercial use, distribution, adaptation, and reproduction provided the original author(s) and source are given appropriate credit.

Received: February 16, 2017

Accepted: March 9, 2017

Citation: US Ophthalmic Review, 2017:10(1):35-8

Corresponding Author: Penny A Asbell, MD, MBA, Cornea Service and Refractive Surgery Center, Icahn School of Medicine at Mount Sinai, 1190 Fifth Avenue, Annenberg Bldg 22-12, New York, NY 10029, US. E: penny.asbell@mssm.edu

Support: The publication of this article was supported by Bausch + Lomb, a division of Valeant Pharmaceuticals International Inc. who were given the opportunity to review the article for scientific accuracy before submission. Any resulting changes were made at the author's discretion.
Antibiotic-resistant bacteria cause infections worldwide, with potentially serious health consequences, ${ }^{1}$ although the majority of such infections involve resistance to systemically administered antibiotics used in the treatment of systemic infections. The first case of methicillin resistance in Staphylococcus aureus (MRSA) was reported in 1961, and was an uncommon finding until the 1990s when community-acquired MRSA became so prevalent in the general population that health authorities considered it endemic. ${ }^{2}$ Although the incidence of healthcare-associated MRSA infections is declining due to preventive hygiene measures in the hospital setting, rates of community-acquired MRSA infections have increased in the general population over the last 10 years. Other pathogens that are resistant to antibiotics include vancomycin-resistant enterococci and multidrug-resistant streptococcus pneumoniae, Mycobacterium tuberculosis, and Neisseria gonorrhoeae. In the US, the Centers for Disease Control and Prevention (CDC) and leading scientists have developed strategies to help reduce antibiotic resistance that include improving diagnosis, tracking and prescribing practices, optimizing therapeutic regimens, adopting antibiotic stewardship programs, and preventing infection transmission.

Ophthalmic bacterial infections are often treated before the causative pathogen is identified, and as such, antibiotic resistance in ocular infections is of particular concern. Whether an infection is on the ocular surface or intraocular, permanent loss of vision may result if appropriate treatment is not initiated promptly, and antibiotic-resistant pathogens can complicate treatment selection. S. aureus, coagulase-negative staphylococcal (CoNS), S. pneumoniae, Pseudomonas aeruginosa, and Haemophilus influenzae are common causes of ocular bacterial 
infections, with both $S$. aureus and CoNS frequently isolated from conjunctivitis, keratitis, endophthalmitis, and blepharitis. ${ }^{3}$

There has been a dramatic rise in the prevalence of methicillin-resistant staphylococci in ophthalmic infections. A study of $S$. aureus isolates from eye infections submitted to The Surveillance Network by more than 200 laboratories in the US from January 2000 to December 2005 showed that the proportion of MRSA increased from $29.5 \%$ in 2000 to $41.6 \%$ in 2005. ${ }^{4}$ Microbial keratitis is recognized as a significant sight-threatening complication that may result from refractive surgery with a reported incidence ranging from $0 \%$ to $1.5 \% .5,6$ Solomon et al. reported an incidence of one infection for every 2,131 refractive surgery procedures in 2004, which increased to an incidence of one infection every 1,102 procedures in 2011; MRSA was the most common organism cultured. ${ }^{5}$ High levels of methicillin resistance were also reported for S. aureus (51.9\%) and $S$. epidermidis (56.0\%) isolates collected from ocular infections between 2003 and 2008 at the Bascom Palmer Eye Institute.? In another study, Gentile et al. reported increasing methicillin resistance among staphylococci in their retrospective study of endophthalmitis cases from 1987 to 2011. Methicillin resistance increased from 18\% for S. aureus and 31\% for Staphylococcus epidermidis in 1987 to 55\% for both in 2011. ${ }^{8}$ MRSA infection and other multidrug-resistant bacterial pathogens can be detrimental to vision and represent a major therapeutic challenge.,39

There is a paucity of studies that monitor antibiotic resistance among ocular bacteria, and this represents a substantial unmet need. This article aims to describe ocular antibiotic resistance surveillance programs in the US, and to present the latest findings of the Antibiotic Resistance Monitoring in Ocular micRoorganisms (ARMOR) study, the only nationwide ongoing surveillance study specific to ocular bacterial pathogens.

\section{Ocular TRUST}

In the past decade, surveillance data have provided clinicians with a large, nationwide picture of resistance and susceptibility patterns in ocular infections. The Tracking Resistance in the United States Today (TRUST) study was developed with the objective of evaluating in vitro susceptibility of bacterial isolates collected yearly from approximately 200 clinical laboratories throughout the US. ${ }^{10}$ At the time of inception, ocular isolates were not a primary consideration although ocular isolates were periodically submitted.

In order to monitor the growing problem of ocular antibiotic resistance, the program was expanded to include an ocular-specific substudy, the Ocular TRUST study. ${ }^{11}$ The Ocular TRUST study objectives were to annually evaluate in vitro antimicrobial susceptibility of $S$. aureus, S. pneumoniae, and $H$. influenzae isolated from ocular infections to fluoroquinolones (ciprofloxacin, gatifloxacin, levofloxacin, moxifloxacin), macrolides (azithromycin), aminoglycosides (tobramycin), penicillins, dihydrofolate reductase inhibitors (trimethoprim), and polypeptides (polymyxin B). The initial study reported on ocular isolates collected from 2005 through 2006 from 35 institutions across the US and found $16.8 \%$ of S. aureus isolates to be methicillin-resistant; intermediate and full resistance to penicillin, azithromycin, and trimethoprim was seen in $18.3 \%, 22.4 \%$, and $22.4 \%$ of pneumococcal isolates, respectively; and no resistance was reported among $H$. influenzae isolates.11

Reports from the second and third years of the study (Ocular TRUST 2 and Ocular TRUST 3) showed an increase in methicillin resistance in S. aureus to almost $50 \%$ by 2008. The Ocular TRUST 2 and Ocular TRUST 3 studies included surveillance of CONS, among which methicillin resistance was found to be $62.0 \%$ in 2008. ${ }^{12,13}$ The investigators concluded that continued antibacterial surveillance of ocular pathogens should be undertaken.

\section{ARMOR}

The multicenter, prospective ARMOR study began in 2009 and was designed to survey antibiotic resistance among S. aureus, CoNS, S. pneumoniae, $H$. influenzae, and $P$. aeruginosa isolates from ocular infections. ARMOR is currently ongoing, and is the only multicenter, national survey of antibiotic resistance patterns among bacterial isolates specific to ophthalmology. ${ }^{14}$

Participating centers (including eye centers and both university and community hospitals) were invited to submit clinically relevant bacterial isolates cultured from both topical and intraocular infections. Bacterial isolates were sent to an independent, central laboratory if they were considered a causal factor in the ocular infection. The antibacterial minimum inhibitory concentrations (MICs; i.e., the lowest concentrations that inhibit growth) were determined for each antibiotic class by broth microdilution using the Clinical and Laboratory Standards Institute (CLSI) procedures with frozen antimicrobial microtiter panels. ${ }^{15}$ Antibiotics tested included fluoroquinolones (ciprofloxacin, moxifloxacin, gatifloxacin, besifloxacin, levofloxacin, and ofloxacin), macrolides (azithromycin), aminoglycosides (tobramycin), lincosamides (clindamycin), cephalosporins (ceftriaxone and ceftazidime), penicillins (oxacillin, penicillin, and piperacillin), carbapenems (imipenem), dihydrofolate reductase inhibitors (trimethoprim), polypeptides (polymyxin B), amphenicols (chloramphenicol), tetracyclines (tetracycline), and glycopeptides (vancomycin). Not all antibiotics were tested in all years of ARMOR. As breakpoints do not exist for topically applied ophthalmic antibiotics, CLSI breakpoints, which are established from in vitro, clinical, and pharmacokinetic pharmacodynamic study data of systemically administered antimicrobial agents, were used (where available) to classify isolates as susceptible, intermediate, or resistant. ${ }^{16}$ Staphylococci were classified as methicillin-resistant or methicillinsusceptible based on susceptibility to oxacillin. Changes in the resistance rate over time were determined using the Cochran-Armitage test for linear trends in proportions. ${ }^{17}$

Data from the first 5 years of ARMOR $^{14}$ for isolates $(n=3,237)$ collected from 2009 through 2013 showed methicillin resistance was common among staphylococcal isolates from ocular infections, but did not increase over the 5 -year study period $(p \leq 0.22)$. Methicillin resistance was found among $493 \mathrm{~S}$. aureus isolates (42.2\%) and 493 CoNS isolates (49.7\%). Methicillin-resistant isolates were likely to be concurrently resistant to fluoroquinolones, aminoglycosides, or macrolides $(p<0.001)$. Multidrug resistance, defined as resistance to three or more antibacterial drug classes, was found in 428 MRSA isolates (86.8\%) and 381 methicillinresistant CONS (MRCONS) isolates (77.3\%). S. pneumoniae showed significant resistance to azithromycin (113 isolates, 34.2\%) and penicillin (105 isolates, 31.8\%), whereas resistance among $P$. aeruginosa and $H$. influenzae was low against the tested antibiotics. Staphylococcal isolates were more likely to be methicillin-resistant if they were collected from elderly patients or from patients in the southern US $(p<0.001)$. Small but significant decreases in resistance were found to some antibiotics over the 5-year study period. Ciprofloxacin resistance decreased among CONS and MRCONS, and tobramycin resistance decreased among CONS $(p \leq 0.03)$. 
Figure 1: Multidrug resistance among ocular staphylococci
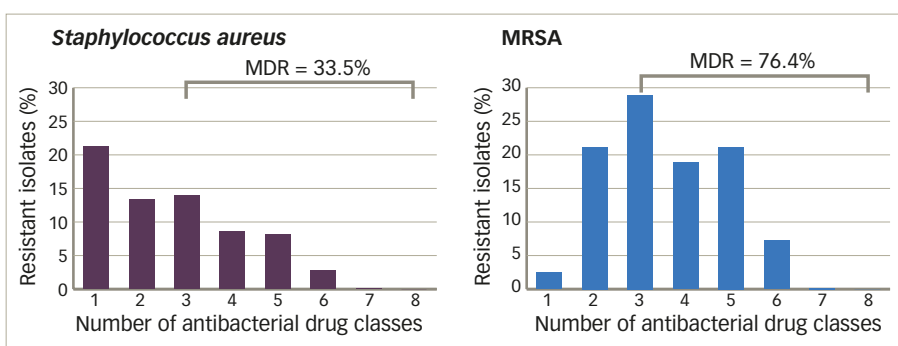

CoNS
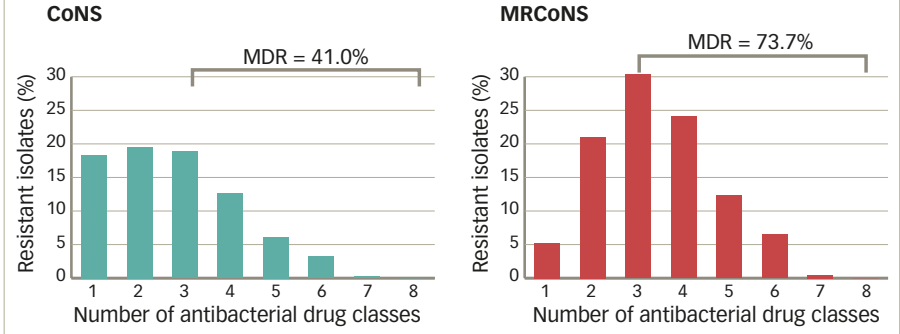

CONS = coagulase-negative staphylococci; $M D R=$ multidrug resistant;

MRCONS = methicillin-resistant CONS; MRSA = methicillin-resistant Staphylococcus aureus.

Results for isolates collected from 2009 through 2015, representing seven years of ARMOR, were presented recently, encompassing a cumulative total of 4,226 ocular isolates from 86 sites in 40 states over the 7 years. ${ }^{18}$ For this larger dataset, the anatomical source was known for 1,956 isolates (46.3\%) and included the conjunctiva ( $n=909)$, cornea ( $n=865)$, aqueous humor $(n=61)$, and vitreous humor $(n=121)$. Overall, resistance rates among ocular bacterial pathogens were comparable to those found over the first 5-year period. ${ }^{14}$ Similar levels of methicillin resistance were observed among staphylococci to those reported for the 5-year dataset. Of 1,483 S. aureus isolates, 567 (38.2\%) were methicillin-resistant whereas among 1,285 CoNS isolates, 632 (49.2\%) were methicillin-resistant. Also, as in the 5-year dataset, the rate of multidrug resistance among methicillin-resistant strains was high with $76.4 \%$ and $73.7 \%$ of MRSA and MRCoNS isolates, respectively, resistant to three or more antimicrobial classes (see Figure 1). However, all staphylococcal isolates were sensitive to vancomycin. Of S. pneumoniae isolates $(n=421)$ in the 7 -year dataset, $36.8 \%$ were resistant to azithromycin and $34.0 \%$ were resistant to penicillin, again consistent with the 5-year dataset. Among $P$. aeruginosa isolates $(n=524)$, low antibiotic resistance was found, and almost no antibiotic resistance was observed among $H$. influenzae isolates $(n=513)$.

When antibiotic resistance rates were evaluated over time, several trends were observed (see Figure 2). Notably, a small but significant decrease in resistance to methicillin (oxacillin) was reported ( $\mathrm{p} \leq 0.001)$ among S. aureus isolates that had not been evident in the 5-year dataset. By contrast, methicillin resistance did not decrease for CoNS over the 7-year time frame. There were also small but significant decreases in resistance of $S$. aureus against azithromycin, ciprofloxacin, and tobramycin ( $\leq 0.001)$; MRSA against tobramycin ( $p=0.022)$; CoNS against ciprofloxacin $(p=0.006)$; and $P$. aeruginosa against ciprofloxacin $(p=0.013)$ and tobramycin $(p=0.019)$. In addition, MRCoNS showed a small but significant $(p=0.021)$ increase in resistance against tobramycin. The largest change over time was in S. pneumoniae, which showed a significant increase ( twofold) in resistance to azithromycin ( $p=0.025)$ over the 7-year study period. ${ }^{18}$ With the exception of the decrease in resistance to ciprofloxacin among
Figure 2: Antibiotic resistance during the seven-year study period
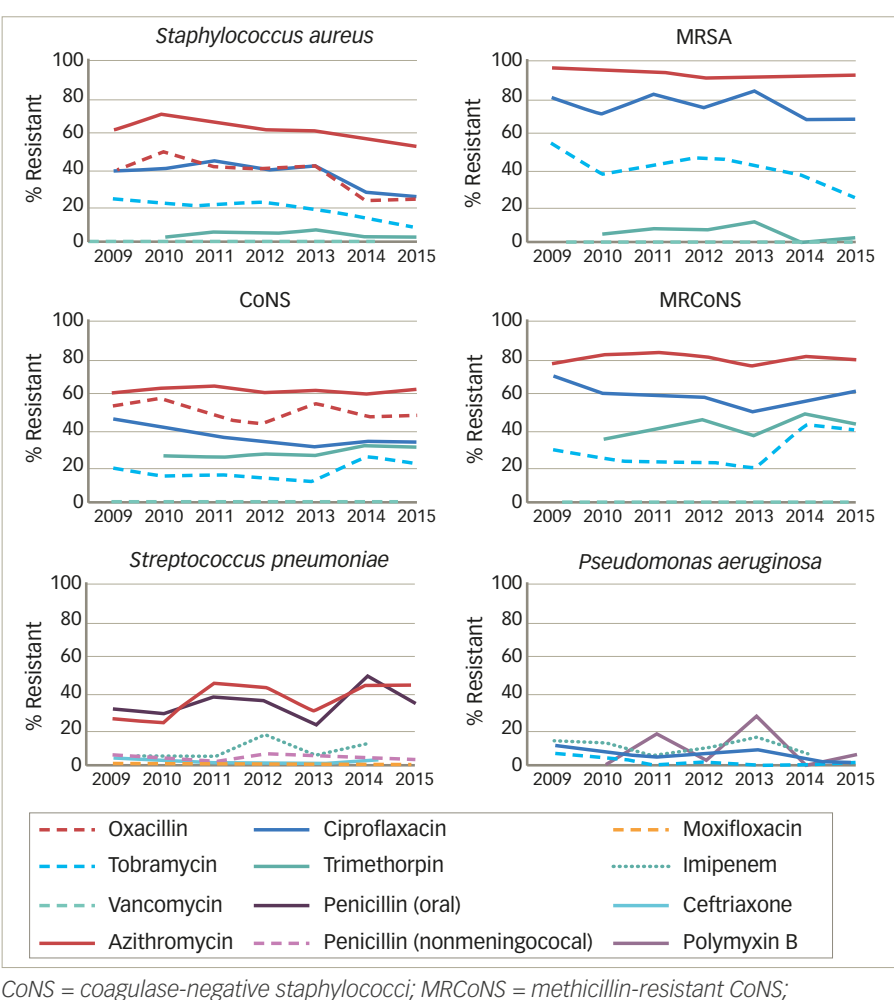

CONS = coagulase-negative staphylococci; MRCoNS = methicillin-resistant CoNS; MRSA = methicillin-resistant Staphylococcus aureus.

CoNS, these changes had not been noted in the 5-year data. While resistance during the 7-year period was notable for several antibiotics, including that for trimethoprim among CoNS and MRCONS, no other trends in resistance over time were noted.

Besifloxacin, a c8-chloro-fluoroquinolone, was developed for topical ophthalmic use only and launched as Besivance (besifloxacin ophthalmic suspension 0.6\%) in the US in 2009. As no systemic formulation exists for besifloxacin, there are no systemic breakpoints available on which to classify bacterial isolates as susceptible, intermediate, or resistant to besifloxacin. ${ }^{16}$ To evaluate the potential efficacy of, or resistance to, besifloxacin, $\mathrm{MIC}_{90}$ data (MIC inhibiting the visible growth of $90 \%$ of all isolates) for besifloxacin was compared with that of other antibacterials, including other fluoroquinolones. Comparisons of cumulative $\mathrm{MIC}_{90}$ values showed wide variations within the fluoroquinolone class of antibiotics, particularly against staphylococci, with newer fluoroquinolones (besifloxacin, moxifloxacin, and gatifloxacin) having a lower $\mathrm{MIC}_{90}$ compared with older fluoroquinolones (ciprofloxacin), and with vancomycin and besifloxacin demonstrating the lowest $\mathrm{MIC}_{90}$ (see Table 1). These differences were most apparent when considering $\mathrm{MIC}_{90} \mathrm{~S}$ against methicillin-resistant staphylococci, suggesting that besifloxacin may have similar clinical activity against these strains to vancomycin, although further studies are needed. Of the antibiotics tested, besifloxacin had the lowest $\mathrm{MIC}_{90}$ for S. pneumoniae, while ciprofloxacin had the lowest $\mathrm{MIC}_{90}$ values for $P$. aeruginosa and $H$. influenzae isolates. There were no changes in besifloxacin $\mathrm{MIC}_{90} \mathrm{~S}$ over the 7-year collection period, suggesting there have been no meaningful changes in bacterial susceptibility to besifloxacin since its launch in 2009. 
Table 1: In vitro $\mathrm{MIC}_{90}$ values $(\mu \mathrm{g} / \mathrm{mL})$ for ocular bacterial isolates

\begin{tabular}{|c|c|c|c|c|c|c|}
\hline \multirow[b]{2}{*}{ Antibiotic } & \multicolumn{3}{|c|}{ Staphylococcus aureus } & \multicolumn{3}{|l|}{ CONS* } \\
\hline & $\begin{array}{l}\text { All } \\
n=1,483\end{array}$ & $\begin{array}{l}\text { MRSA } \\
n=567\end{array}$ & $\begin{array}{l}\text { MSSA } \\
\mathrm{n}=916\end{array}$ & $\begin{array}{l}\text { All } \\
n=1,285\end{array}$ & $\begin{array}{l}\text { MRCONS } \\
n=632\end{array}$ & $\begin{array}{l}\text { MSCONS } \\
n=653\end{array}$ \\
\hline Vancomycin & 1 & 1 & 1 & 2 & 2 & 2 \\
\hline Besifloxacin & 1 & 2 & 0.25 & 2 & 4 & 0.25 \\
\hline Moxifloxacin & 8 & 16 & 1 & 16 & 32 & 1 \\
\hline Gatifloxacin & 8 & 16 & 2 & 16 & 32 & 1 \\
\hline Ciprofloxacin & 128 & 256 & 8 & 64 & 64 & 8 \\
\hline Trimethoprim & 2 & 2 & 2 & $>128$ & $>128$ & 64 \\
\hline Tobramycin & 256 & 256 & 1 & 8 & 32 & 4 \\
\hline Azithromycin & $>512$ & $>512$ & $>512$ & $>512$ & $>512$ & $>512$ \\
\hline Antibiotic & \multicolumn{2}{|c|}{$\begin{array}{l}\text { Streptococcus } \\
\text { pneumoniae } n=421\end{array}$} & \multicolumn{2}{|c|}{$\begin{array}{l}\text { Pseudomonas } \\
\text { aeruginosa } n=524\end{array}$} & \multicolumn{2}{|c|}{$\begin{array}{l}\text { Haemophilus } \\
\text { influenzae } n=513\end{array}$} \\
\hline Besifloxacin & \multicolumn{2}{|l|}{0.06} & \multicolumn{2}{|l|}{4} & \multicolumn{2}{|l|}{0.03} \\
\hline Moxifloxacin & \multicolumn{2}{|l|}{0.12} & \multicolumn{2}{|l|}{4} & \multicolumn{2}{|l|}{0.03} \\
\hline Gatifloxacin & \multicolumn{2}{|l|}{0.25} & \multicolumn{2}{|l|}{2} & \multicolumn{2}{|l|}{0.015} \\
\hline Ciprofloxacin & \multicolumn{2}{|l|}{1} & \multicolumn{2}{|l|}{0.5} & \multicolumn{2}{|l|}{0.015} \\
\hline Tobramycin & \multicolumn{2}{|l|}{32} & \multicolumn{2}{|l|}{1} & \multicolumn{2}{|l|}{2} \\
\hline Azithromycin & \multicolumn{2}{|l|}{$>128$} & \multicolumn{2}{|l|}{256} & \multicolumn{2}{|l|}{2} \\
\hline Penicillin (oral) & \multicolumn{2}{|l|}{1} & \multicolumn{2}{|l|}{ NT } & \multicolumn{2}{|l|}{$>4$} \\
\hline
\end{tabular}

*Includes 972 Staphylococcus epidermidis isolates. CoNS = coagulase-negative staphylococci: MRCONS = methicillin-resistant CONS; MRSA = methicillin-resistant Staphylococcus aureus; MSCONS = methicillin-susceptible CONS; MSSA = methicillinsusceptible S. aureus; NT = not tested.

\section{Conclusions}

Limitations of the ARMOR surveillance study include the potential for sampling bias owing to the infrequent practice of culturing bacterial pathogens, and the application of systemic breakpoints to define isolates as susceptible or resistant to ocular treatment. ${ }^{14}$ Systemic breakpoints are derived from pharmacokinetic and pharmacodynamic studies of systemic infections, and their value when assessing the susceptibility of ocular isolates exposed to topically applied antibiotics remains unclear. However, in the absence of topical, ophthalmic breakpoints, systemic breakpoints remain a useful means of determining the antibiotic resistance status of isolates from ophthalmic infections. Another consideration is that while antibiotic concentration in external ocular tissue upon topical application may exceed the MIC needed for common ocular isolates, topical antibiotics are rapidly eliminated from tears due to tear turnover.

As with their systemic counterparts, ocular pathogens are demonstrating high in vitro resistance to antimicrobial agents. Vancomycin remains effective in vitro against staphylococcal isolates, but it is not commercially available as a topical formulation and must be compounded for use. Although newer drugs, such as besifloxacin, demonstrate significant in vitro activity against many resistant bacterial pathogens, the resistance of staphylococcal isolates to older fluoroquinolones is cause for concern. The fact that methicillin resistance is decreasing among $S$. aureus, is, however, a promising finding that may suggest an increased awareness of the problems of antibiotic resistance and also improved antibiotic stewardship. Indeed, in terms of systemic infections, increased education regarding judicious antibiotic use by limiting overprescription of antibiotics, cycling between antibiotic classes, and using combination therapies has led to a decrease in antibiotic resistance in systemic infections. ${ }^{19-22}$

The ARMOR study is the only nationwide evaluation of antibacterial susceptibility of common ocular pathogens in the US, and now includes almost 1,000 additional isolates beyond those included in the 2015 evaluation. ${ }^{18}$ Although trends over the past 7 years show a small but significant decrease in $S$. aureus resistance to some antibiotic classes, and confirmed the previous finding of a decrease in resistance among CoNS to ciprofloxacin, some increases in resistance were also noted. For instance, small but significant increases in resistance were noted among MRCoNS to tobramycin and among S. pneumoniae to azithromycin. Together these findings underscore the need for continued surveillance of antibiotic resistance trends and to help guide treatment decisions for ocular bacterial infections. $\square$
1. Ventola $\mathrm{CL}$, The antibiotic resistance crisis: part 2: management strategies and new agents, PT, 2015;40:344-52.

2. Speller DC, Johnson AP, James D, et al., Resistance to methicillin and other antibiotics in isolates of Staphylococcus aureus from blood and cerebrospinal fluid, England and Wales, 1989-95, 1ancet 1997:350:323-5.

3. Kowalski RP, Dhaliwal DK, Ocular bacterial infections: current and future treatment options, Expert Rev Anti Infect Ther, 2005;3:131-9.

4. Asbell PA, Sahm DF, Shaw M, et al., Increasing prevalence of methicillin resistance in serious ocular infections caused by Staphylococcus aureus in the United States: 2000 to 2005 J Cataract Refract Surg, 2008;34:814-8.

5. Solomon R, Donnenfeld ED, Holland EJ, et al., Microbial keratitis trends following refractive surgery: results of the ASCRS infectious keratitis survey and comparisons with prior ASCRS surveys of infectious keratitis following keratorefractive procedures, J Cataract Refract Surg, 2011;37:1343-50.

6. Stulting RD, Carr JD, Thompson KP, et al., Complications of laser in situ keratomileusis for the correction of myopia, Ophthalmology, 1999:106:13-20.

7. Miller D, Chang JS, Flynn HW, et al., Comparative in vitro susceptibility of besifloxacin and seven comparators against ciprofloxacin- and methicillin-susceptible/nonsusceptible staphylococci, I Ocul Pharmacol Ther, 2013;29:339-44.

8. Gentile RC, Shukla S, Shah M, et al., Microbiological spectrum and antibiotic sensitivity in endophthalmitis: a 25-year review, Ophthalmology, 2014;121:1634-42.

9. Ni N, Nam EM, Hammersmith KM, et al., Seasonal, geographic, and antimicrobial resistance patterns in microbial keratitis: 4-year experience in eastern Pennsylvania, Cornea, 2015;34:296-302

10. Thornsberry C, Jones ME, Hickey ML, et al., Resistance surveillance of Streptococcus pneumoniae, Haemophilus influenzae and Moraxella catarrhalis isolated in the United States, 1997-1998, Moraxella catarrhalis Isolated in the United

11. Asbell PA, Colby KA, Deng S, et al., Ocular TRUST: nationwide antimicrobial susceptibility patterns in ocular isolates, Am J Ophthalmol, 2008;145:951-8.

12. Asbell PA, Sahm DF, Longitudinal nationwide antimicrobial susceptibility surveillance in ocular isolates: results from Ocular TRUST 2, Presented at: American Society of Cataract and Refractive Surgery Annual Meeting: April 28, 2008; San Diego, CA

13. Asbell PA, Sahm DF, Shedden A, Ocular TRUST 3: ongoing longitudinal surveillance of antimicrobial susceptibility in ocular isolates. Presented at: American Society of Cataract and Refractive Surgery Annual Meeting; April 7, 2009; San Francisco, CA.

14. Asbell PA, Sanfilippo CM, Pillar CM, et al., Antibiotic Resistance Among Ocular Pathogens in the United States: Five-Year Results From the Antibiotic Resistance Monitoring in Ocular Microorganisms (ARMOR) Surveillance Study, JAMA Ophthalmol, 2015:133:1445-54.

15. CLSI, Clinical and Laboratory Standards Institute. Methods for Dilution Antimicrobial Susceptibility Tests for Bacteria That Grow Aerobically: Approved Standard. Wayne, PA: Clinical \& LaboratoryStandards Institute; 2009-2015. CLSI documents M7-A8 through M7-A10.

16. CLSI, Clinical and Laboratory Standards Institute. Performance
Standards for Antimicrobial Susceptibility Testing. Wayne, PA Clinical \& Laboratory Standards Institute; 2009-2015. CLSI documents M100-S19 through M100-S25.

17. Armitage $P_{1}$, Tests for linear trends in proportions and frequencies, Biometrics, 1955:11:375-86.

18. Melton $R$, Thomas $R$, Sanfilippo $C M$, et al., Antibiotic resistance trends among ocular pathogens in the United States: Seven-year cumulative results from the ARMOR surveillance study. Presented at the annual meeting of the American Academy of Optometry, November 912 2016, Anaheim, CA.

19. Brinsley K, Srinivasan A, Sinkowitz-Cochran R, et al., Implementation of the Campaign to Prevent Antimicrobial Resistance in Healthcare Settings: 12 Steps to Prevent Antimicrobial Resistance Among Hospitalized Adults experiences Antimicrobial Resistance Among Hospitalized Adults exp

20. Stone SP, Fuller $C$, Savage J, et al., Evaluation of the national Cleanyourhands campaign to reduce Staphylococcus aureus bacteraemia and Clostridium difficile infection in hospitals in England and Wales by improved hand hygiene: four year, prospective, ecological, interrupted time series study, $B M \perp$, 2012;344:e3005.

21. DiDiodato $G$, McArthur L. Evaluating the Effectiveness of an Antimicrobial Stewardship Program on Reducing the Incidence Rate of Healthcare-Associated Clostridium difficile Infection: A Non-Randomized, Stepped Wedge, Single-Site, Observational Study, PLoS One, 2016;11:e0157671.

22. Davey P, Brown E, Charani E, et al., Interventions to improve antibiotic prescribing practices for hospital inpatients, Cochrane Database Syst Rev, 2013;CD003543. 